\title{
Remarks on a conjecture of Barát and Tóth
}

\author{
Atílio G. Luiz* \\ Institute of Computing \\ University of Campinas \\ Campinas, São Paulo, Brazil \\ gomes.atilio@gmail.com
}

\author{
R. Bruce Richter ${ }^{\dagger}$ \\ Combinatorics \& Optimization \\ University of Waterloo \\ Waterloo, Ontario, Canada \\ brichter@uwaterloo.ca
}

Submitted: May 20, 2013; Accepted: Mar 5, 2014; Published: Mar 17, 2014

Mathematics Subject Classifications: 05C15, 05C10

\begin{abstract}
In 2010, Barát and Tóth verified that any $r$-critical graph with at most $r+4$ vertices has a subdivision of $K_{r}$. Based in this result, the authors conjectured that, for every positive integer $c$, there exists a bound $r(c)$ such that for any $r$, where $r \geqslant r(c)$, any $r$-critical graph on $r+c$ vertices has a subdivision of $K_{r}$. In this note, we verify the validity of this conjecture for $c=5$, and show counterexamples for all $c \geqslant 6$.
\end{abstract}

Keywords: colour-critical graphs; Hajós conjecture; Albertson conjecture

\section{Introduction}

In this note, we discuss a few points arising from the interesting paper of Barát and Tóth [2]. Their motivation is Albertson's Conjecture that if the chromatic number $\chi(G)$ of a graph $G$ is $r$, then the crossing number $\operatorname{cr}(G)$ of $G$ is at least that of $K_{r}$. In other words, among all graphs with chromatic number at least $r$, the one with smallest crossing number is $K_{r}$. This is trivial for $r \leqslant 4$ and follows from the Four Colour Theorem for $r=5$. It was proved by Albertson, Cranston, and Fox [1] for $r \leqslant 12$ (precisely the values of $r$ for which $\operatorname{cr}\left(K_{r}\right)$ is currently known). Barát and Tóth extended this to $r \leqslant 16$ by using their new lower bound on crossing numbers to show that every $r$-chromatic graph other than $K_{r}$ has crossing number at least the conjectured value of $\operatorname{cr}\left(K_{r}\right)$.

Albertson's Conjecture is related to Hajós' Conjecture that every $r$-chromatic graph contains a subdivision of $K_{r}$. The Hajós Conjecture obviously implies the Albertson

*Supported by FAPESP grant 12/16418-1.

${ }^{\dagger}$ Supported by NSERC. 
Conjecture. Unfortunately, the Hajós Conjecture is false for $r \geqslant 7$ [3] and remains open for $r=5,6$. There is more detailed information to be found in [8].

Barát and Tóth were interested in graphs that satisfy the Hajós Conjecture. Recall that a graph $G$ is $r$-critical if $\chi(G)=r$ but, for every edge $e$ of $G, \chi(G-e)<r$. Dirac [5, 7] proved that no $r$-critical graph has $r+1$ vertices and that $C_{5} \vee K_{r-3}$ is the only $r$-critical graph with $r+2$ vertices. (Here $G \vee H$ is the join of $G$ and $H$; it is the graph obtained from the disjoint union of $G$ and $H$ by adding all edges having one end in $G$ and one end in $H$.) Gallai [9] extended this by showing that, for $r \geqslant 4$, there are only two $r$-critical graphs with $r+3$ vertices.

An observation of Barát and Tóth is that, for $r \geqslant 6$, there are precisely $22 r$-critical graphs with $r+4$ vertices. In the next section, we prove the following, a straightforward generalization of Barát and Tóth's observation.

Theorem 1. Let $c$ be a positive integer. There are numbers $n(c)$ and $r(c)$ so that, for any $r \geqslant r(c)$, there are precisely $n(c) r$-critical graphs with $r+c$ vertices.

In particular, $r(5)=7$ and $n(5)=395$. Moreover, every $r$-critical graph with $r+5$ vertices has a subdivision of $K_{r}$.

Barát and Tóth also proposed the following conjecture.

Conjecture 2. For every positive integer $c$, there is a number $r(c)$ so that, if $r \geqslant r(c)$, then any $r$-critical graph with $r+c$ vertices has a subdivision of $K_{r}$.

Theorem 1 shows this conjecture is true for $c=5$. We shall see in the next section that the methods used to prove Theorem 1 combine with some standard examples to demonstrate that this conjecture is not true for any $c \geqslant 6$.

The note concludes with some remarks on the assertion of Barát and Tóth that the Catlin examples satisfy the Albertson Conjecture. We explain why their proof is not valid, and so it is still open whether the Catlin examples satisfy the Albertson Conjecture.

\section{Results}

This section contains the proof of Theorem 1 and the proof of Conjecture 2 for $c=5$, along with its refutation for every $c \geqslant 6$. The main theoretical tool is the following result of Gallai [9]. A vertex $v$ in a graph $G$ is universal if $v$ is adjacent to every other vertex in $G$.

Theorem 3 (Gallai [9]). Let $G$ be an $r$-critical, $n$-vertex graph with $r \geqslant 3$. Then $G$ contains at least $\left\lceil\frac{3}{2}\left(\frac{5}{3} r-n\right)\right\rceil$ universal vertices. In particular, if $r>\frac{3}{2} c$, then any $r$ critical graph with $r+c$ vertices has a universal vertex.

We are now ready for the proof of Theorem 1 .

Proof of Theorem 1. Theorem 3 implies that, for $r>(3 c) / 2$, an $r$-critical graph with $r+c$ vertices has a universal vertex. Since it is well-known that $G \vee v$ is $(r+1)$-critical 
if and only if $G$ is $r$-critical, for $r>(3 c) / 2$, the number of $r$-critical graphs with $r+c$ vertices is the same as the number of $(r-1)$-critical graphs with $(r-1)+c$ vertices; thus, $r(c) \leqslant\lfloor(3 c) / 2\rfloor$ and $n(c)$ is the number of $(\lfloor(3 c) / 2\rfloor)$-critical graphs with $\lfloor(3 c) / 2\rfloor+c$ vertices. In particular, $r(5) \leqslant 7$.

For the second part of Theorem 1, we consider the special case $c=5$. To see that $n(5)=395$, Royle's table [13] of small critical graphs shows there are exactly the following graphs that are, for some $r, r$-critical, with $r+5$ vertices, and have no universal vertex:

(i) twenty-one 4-critical graphs on 9 vertices;

(ii) one hundred and forty-one 5-critical graphs on 10 vertices;

(iii) two hundred and thirty-one 6-critical graphs on 11 vertices; and

(iv) two 7-critical graphs on 12 vertices.

Because $r(5) \leqslant 7$, the two 7-critical graphs in this list show $r(5)=7$. For $r \geqslant 8$, every $r$-critical graph with $r+5$ vertices has a universal vertex and so is the join of some $K_{s}$ with one of the 395 listed graphs. That is, $n(5)=395$.

Moreover, it suffices to show that each one of the 395 graphs in the list has a subdivision of the appropriate $K_{r}$. Dirac [6] proved the Hajós Conjecture for $r=4$, so each one of the twenty-one 4-critical graphs of the list has a subdivision of $K_{4}$. For $r=5$, Mader's Theorem [11] that any graph with $n$ vertices and at least $3 n-5$ edges has a subdivision of $K_{5}$ is helpful. For each one of the one hundred and forty-one 5-critical graphs of the list we counted its edges from the adjacency list provided by Royle's table. We checked by hand those graphs with fewer than $3 n-5$ edges; every one had a subdivision of $K_{5}$. Finally, all the two hundred and thirty-one 6-critical graphs were checked by hand, and for each one a $K_{6}$-subdivision was found. Most of the 6-critical graphs have approximately the same structure, which made them less difficult to verify. A typical example is shown in Figure 1. This is Graph 391 in the table. (Royle (personal communication) independently verified by computer program that these 395 graphs satisfy the Hajós Conjecture.)

We now turn our attention to showing that Conjecture 2 is false for every $c \geqslant 6$. In order to do this, we use the following subgraphs of Catlin's graphs $L\left({ }_{k} C_{5}\right)$ [3].

Family $\mathbf{F}_{\mathbf{c}}$. For $c \geqslant 6$, let $F_{c}$ be the graph whose vertex set consists of five non-empty pairwise disjoint sets $A_{1}, A_{2}, C_{1}, C_{2}$, and $C_{3}$ where $\left|C_{1}\right|=\left|C_{2}\right|=\left|C_{3}\right|=3$ and $\left|A_{1}\right|=$ $\left|A_{2}\right|=c-4$, such that these sets induce cliques in $F_{c}$. The sets $A_{1}, A_{2}, C_{1}, C_{2}$, and $C_{3}$ are joined in the following way: $A_{1} \vee C_{1}, A_{1} \vee C_{2}, C_{1} \vee A_{2}, A_{2} \vee C_{3}$, and $C_{2} \vee C_{3}$. Figure 2 shows a scheme of the graphs $F_{c}$.

In the following, we prove that, for $c \geqslant 6, F_{c}$ is a $(c+1)$-critical graph that does not contain a subdivision of $K_{(c+1)}$. Since $F_{c}$ has $2 c+1$ vertices and is $(c+1)$-critical, the join $F_{c} \vee K_{t}$ has $2 c+t+1$ vertices, is $(c+t+1)$-critical, and does not contain a subdivision of $K_{(c+t+1)}$. Thus, Conjecture 2 is false for every $c \geqslant 6$. 

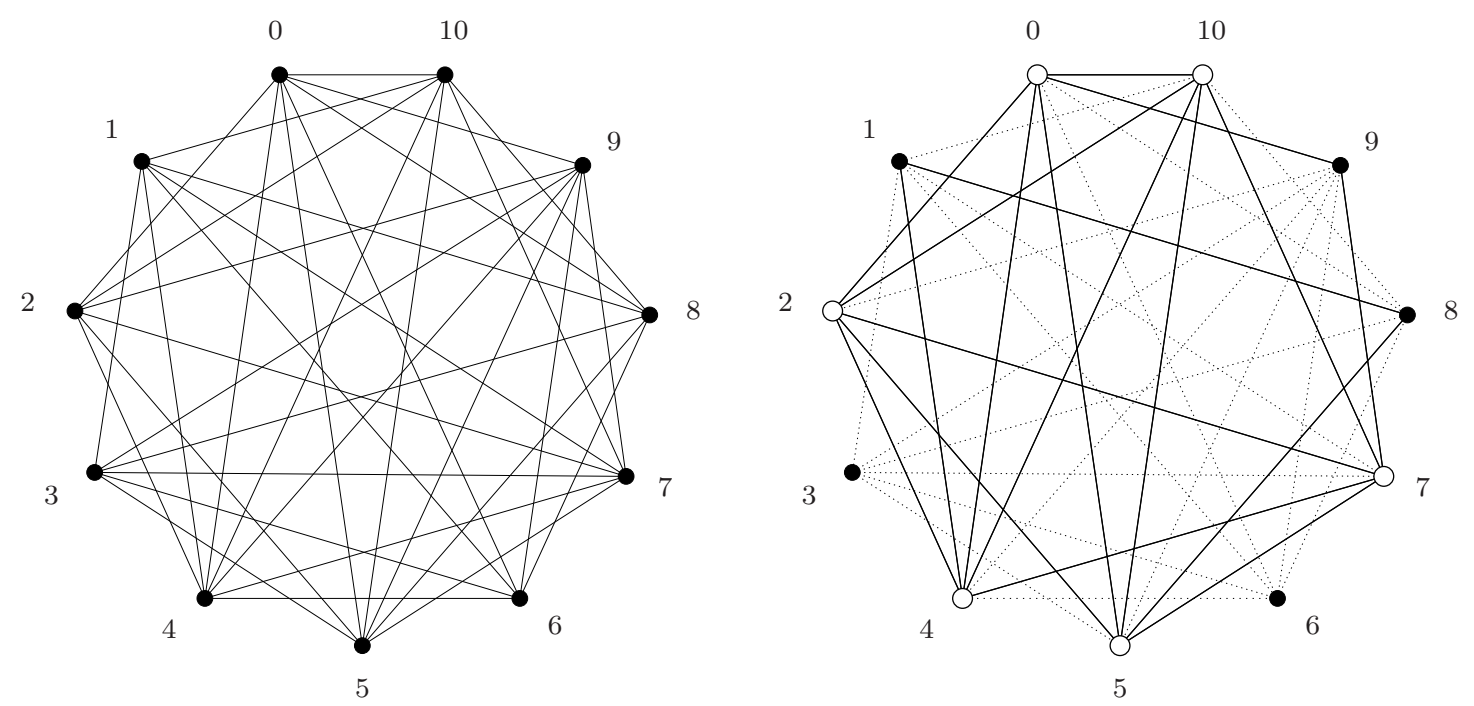

Figure 1: A 6-critical graph and a subdivision of $K_{6}$ in it.

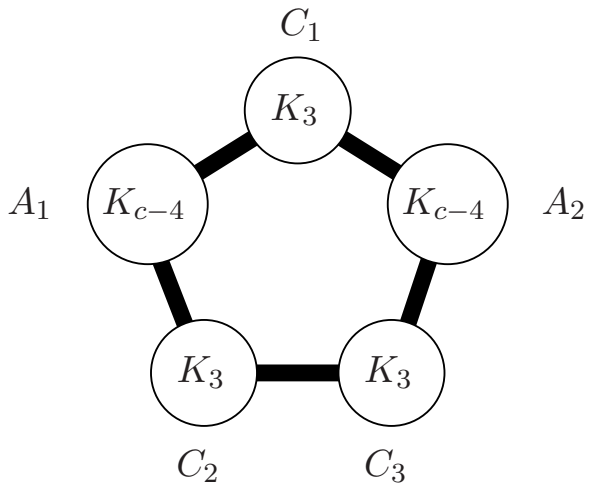

Figure 2: Scheme of a graph $F_{c}$. Heavy edges indicate that every vertex in one set is adjacent to every vertex in the other.

Proposition 4. For $c \geqslant 6, \chi\left(F_{c}\right)=c+1$.

Proof. Since every independent set in $F_{c}$ has at most two vertices, we see that

$$
\chi\left(F_{c}\right) \geqslant \frac{\left|V\left(F_{c}\right)\right|}{2}=\frac{2 c+1}{2}=c+\frac{1}{2} .
$$

For $c \geqslant 6$, the graph $F_{c}$ can be assigned a $(c+1)$-coloring such that the vertices in the sets $A_{1}, A_{2}, C_{1}, C_{2}$, and $C_{3}$ receive the following colors: $C_{1}=\{1,2,3\}, C_{2}=\{1,4,5\}$, $C_{3}=\{2,6,7\}, A_{1}=\{6,7,8,9, \ldots, c+1\}$, and $A_{2}=\{4,5,8,9, \ldots, c+1\}$.

Proposition 5. For $c \geqslant 6, F_{c}$ is $(c+1)$-critical.

Proof. For $c \geqslant 6$, we show that, after the removal of an arbitrary edge $e$, the graph $F_{c}-e$ is $c$-colorable. There are 6 different cases to consider. 
Case 1. $e \in E\left(C_{1}\right)$.

For $c \geqslant 6$, the vertex subsets of $F_{c}-e$ can be assigned the following colors: $C_{1}=\{1,2\}$, $C_{2}=\{1,6,7\}, C_{3}=\{2,4,5\}, A_{1}=\{4,5,8, \ldots, c+1\}$, and $A_{2}=\{6,7,8, \ldots, c+1\}$. Observe that the color 3 does not appear in this coloring. Therefore, $F_{c}-e$ is colorable with $c$ colors.

Case 2. Edge $e$ has one endpoint in the set $C_{1}$ and the other endpoint in the set $A_{1}$.

For $c \geqslant 6$, the vertex subsets of $F_{c}-e$ can be assigned the following colors: $C_{1}=$ $\{1,2,3\}, C_{2}=\{2,6,7\}, C_{3}=\{1,3,5\}, A_{1}=\{1,5,8, \ldots, c+1\}$, and $A_{2}=\{6,7,8, \ldots, c+$ $1\}$. Observe that the color 4 does not appear in this coloring. Therefore, $F_{c}-e$ is colorable with $c$ colors.

The remaining cases, listed below, are treated analogously.

Case 3. $e \in E\left(A_{1}\right)$.

Case 4. Edge $e$ has one endpoint in the set $A_{1}$ and the other endpoint in the set $C_{2}$.

Case 5. $e \in E\left(C_{2}\right)$.

Case 6. Edge $e$ has one endpoint in the set $C_{2}$ and the other endpoint in the set $C_{3}$.

The next proposition completes our analysis of the graph $F_{c}$.

Proposition 6. For $c \geqslant 6, F_{c}$ does not contain a subdivision of $K_{(c+1)}$.

Proof. Catlin [3] showed that the graphs $F_{6}$ and $F_{7}$ do not have a subdivision of $K_{7}$ and $K_{8}$, respectively. Thus, we may suppose $c \geqslant 8$ and $F_{c}$ has a subdivision of $K_{c+1}$. Let $W$ be the set of vertices with degree $c$ in a subdivision of $K_{c+1}$. Within the subdivision, any two vertices of $W$ are joined by $c$ pairwise internally-disjoint paths.

Only if $c=8$ does $C_{1} \cup C_{2} \cup C_{3}$ have enough vertices to contain $W$. In this case, $W=V\left(C_{1} \cup C_{2} \cup C_{3}\right)$ and there must be nine disjoint paths through $A_{1}$ representing the edges from $C_{1}$ to $C_{2}$. Since $A_{1}$ has only four vertices, this is impossible.

Thus, in all cases, at least one vertex of $W$ is in $A_{1} \cup A_{2}$. Since $V\left(C_{1} \cup C_{2}\right)$ is a cut-set in $F_{c}$ of size $6<c$, there cannot be vertices of $W$ in both $V\left(A_{1}\right)$ and $V\left(A_{2}\right)$. Therefore, we may assume $W \cap V\left(A_{1}\right) \neq \emptyset$ and $W \cap V\left(A_{2}\right)=\emptyset$.

Since $V\left(C_{1} \cup C_{2}\right)$ also separates $A_{1}$ from $C_{3}$, we deduce that $W \cap V\left(C_{3}\right)=\emptyset$. Therefore, $W \subseteq V\left(C_{1} \cup A_{1} \cup C_{2}\right)$.

Since $|W|=c+1$ and $\left|V\left(C_{1} \cup A_{1} \cup C_{2}\right)\right|=c+2$, exactly one vertex $v$ in $C_{1} \cup A_{1} \cup C_{2}$ is not in $W$. It follows that there are at least 6 (5 is enough) internally-disjoint $C_{1} C_{2}$-paths in $K_{c+1}$ representing the edges from $W \cap V\left(C_{1}\right)$ to $W \cap V\left(C_{2}\right)$. Since at most one vertex of $A_{1}$ is not in $W$, at most one of the $6 C_{1} C_{2}$-paths can go through $A_{1}$. In the other direction, at most three can go through $C_{3}$. Thus there are too few paths for $F_{c}$ to have a subdivision of $K_{c+1}$.

To conclude the proof, there is a subdivision of $K_{c}$ consisting of the $c-4$ vertices in $A_{1}$, three vertices in $C_{1}$, and one vertex in $C_{2}$. This uses only three internally disjoint $C_{1} C_{2}$-paths through $C_{3} \cup A_{2}$. 


\section{Catlin graphs and Albertson's Conjecture}

In their paper, Barát and Tóth claim (Lemma 16) that Catlin's graphs satisfy Albertson's Conjecture. In the proof, they use (asymptotic versions of) the not-yet proved conjectures on the values of the crossing numbers of $K_{2 k}, K_{k}$, and $K_{k, k}$. We prove the following, without any assumption about the crossing number of $K_{k}$ and $K_{k, k}$.

Theorem 7. The graph $F_{c}$ has crossing number at least that of $K_{c+1}$.

Proof. We have already exhibited a subdivision of $K_{c}$ in $F_{c}$. This subdivision uses three internally disjoint $C_{2} C_{1}$-paths through $A_{2}$, but is otherwise disjoint from $A_{2}$. It is known $[10,12]$ that the crossing number of $K_{c}$, denoted $\operatorname{cr}\left(K_{c}\right)$, is at most

$$
f(c)=\frac{1}{4}\left\lfloor\frac{c}{2}\right\rfloor\left\lfloor\frac{c-1}{2}\right\rfloor\left\lfloor\frac{c-2}{2}\right\rfloor\left\lfloor\frac{c-3}{2}\right\rfloor .
$$

Since, $\operatorname{cr}\left(K_{c}\right) \leqslant f(c)$, there is a vertex $v$ of $K_{c}$ so that the edges incident with $v$ are involved in at most $\lfloor 4 f(c) / c\rfloor$ crossings.

Let $D$ be a drawing of $K_{c}$ with at most $f(c)$ crossings. We obtain a drawing $D^{\prime}$ of $K_{c+1}$ by adding a new vertex $v^{\prime}$ close to $v$ and joined to $v$ with no crossings. For each other vertex $w$ of $K_{c}$, we add the edge $v^{\prime} w$ alongside $v w$. These latter edges cross the same number of edges to $K_{c}-v$ as those incident with $v$ do, which is to say at most $\lfloor 4 f(c) / c\rfloor$ crossings. In addition, Woodall [14] observes that the edges at $v^{\prime}$ can be drawn so as to cross the edges incident with $v$ at most $\lfloor(c-1) / 2\rfloor\lfloor(c-2) / 2\rfloor$ times. Thus,

$$
\operatorname{cr}\left(K_{c+1}\right) \leqslant \operatorname{cr}\left(K_{c}\right)+\left\lfloor\frac{4 f(c)}{c}\right\rfloor+\left\lfloor\frac{c-1}{2}\right\rfloor\left\lfloor\frac{c-2}{2}\right\rfloor .
$$

On the other hand, the crossing number of $K_{k}$ is known [4] to be at least $0.8594 f(k)$. The three internally disjoint paths through $A_{2}$ are all incident with a common node of the subdivision of $K_{c}$, so no crossing between two of them is counted in the crossings of this $K_{c}$. Therefore, all the crossings in the $K_{c-1}$ induced by $A_{2} \cup C_{3}$ are additional. We deduce that the crossing number of $F_{c}$ is at least $\operatorname{cr}\left(K_{c}\right)+0.8594 f(c-1)$.

It follows that, if

$$
.8594 f(c-1) \geqslant\left\lfloor\frac{4 f(c)}{c}\right\rfloor+\left\lfloor\frac{c-1}{2}\right\rfloor\left\lfloor\frac{c-2}{2}\right\rfloor,
$$

then the crossing number of $F_{c}$ is at least that of $K_{c+1}$. We note that, since the left side is degree 4 in $c$ while the right is degree 3 in $c(f(c)$ is degree 4 in $c)$, this certainly is true for $c$ large enough. In fact, this holds for $c \geqslant 12$. For $c<12$, already [1] shows that Albertson's Conjecture is true, so in these cases also, $\operatorname{cr}\left(F_{c}\right) \geqslant \operatorname{cr}\left(K_{c+1}\right)$.

Unfortunately, a straightforward analogue of this argument does not show that the Catlin graphs satisfy the Albertson Conjecture. 


\section{Acknowledgments}

We are grateful to Gordon Royle for his insights and computer skills. We also appreciate the suggestions from the referees to improve the presentation.

\section{References}

[1] M. O. Albertson, D. W. Cranston, and J. Fox. Crossings, colorings, and cliques. Electronic Journal of Combinatorics, 16:\#R45, 2009.

[2] J. Barát and G. Tóth. Towards the Albertson conjecture. Electronic Journal of Combinatorics, 17:\#R73, 2010.

[3] P. A. Catlin. Hajós' graph-coloring conjecture: variations and counterexamples. Journal of Combinatorial Theory, Series B, 26:268-274, 1979.

[4] E. de Klerk, D. V. Pasechnik, and A. Schrijver, Reduction of symmetric semidefinite programs using the regular *-representation. Mathematical Programming: Series B, 109(2):613-624, 2007.

[5] G. A. Dirac. Map colour theorems. Canadian Journal of Mathematics, 4:480-490, 1952.

[6] G. A. Dirac. A property of 4-chromatic graphs and some remarks on critical graphs. Journal of the London Mathematical Society, 27:85-92, 1952.

[7] G. A. Dirac. Map colour theorems related to the Heawood colour formula. Journal of the London Mathematical Society, 31:460-471, 1956.

[8] P. Erdős and S. Fajtlowicz. On the conjecture of Hajós. Combinatorica, 1:141-143, 1981.

[9] T. Gallai. Kritische Graphen. II. (German). Magyar Tud. Akad. Mat. Kutat Int. Közl, (8):373-395, 1963.

[10] R. K. Guy. Crossing numbers of graphs, in Graph Theory and Applications, Lecture Notes in Mathematics, 303:111-124, 1972.

[11] W. Mader. 3n- 5 edges do force a subdivision of $K_{5}$. Combinatorica, 18(4):569-595, 1998.

[12] R. B. Richter and C. Thomassen. Relations between crossing numbers of complete and complete bipartite graphs. The American Mathematical Monthly, 104(2):131$137,1997$.

[13] G. Royle. Small graphs.

http://school . maths. uwa.edu. au/ gordon/remote/graphs/.

[14] D. R. Woodall. Cyclic-order graphs and Zarankiewicz's crossing-number conjecture. Journal of Graph Theory, 17(6):657-671, 1993. 